\title{
Ein soziologischer Kongress im Zwischen
}

\section{Von Kurt Georg Rachlitz}

\section{Disproportionale Verhältnisse}

In der Besprechung seines Buches Symbolische Formung am Mittwochnachmittag beschrieb Hans-Georg Soeffner das Ritual eines Trauerzuges. Setzt sich dieser in Bewegung, so steigt die Trauer derer, die sich nahe um den Sarg herum versammeln, schlagartig an. Es lässt sich aber auch Folgendes beobachten: Je weiter die Teilnehmer vom Sarg entfernt sind (mag der Verstorbene zu Lebzeiten sich auch noch so großer Beliebtheit erfreut haben), desto besser wird die Stimmung. Man trifft und unterhält sich.

Ein ähnliches Bild liefert der DGS-Kongress 2012 in Bochum (und Dortmund). Während sich der Titel, die raren Zeitungsberichte, diverse Vorwörter des Hauptprogramms und viele Vorlesungen - d.h. all das, was sich sichtbar gibt - mit nötigem Ernst dem Thema ,Vielfalt und Zusammenhalt' in einem durchaus konventionellen und bisweilen proto-politischen Sinne zuwenden, bröckelt diese prästabilisierte Harmonie an den Rändern (besser: in den Kellern) des Kongresses. Dort finden die Ad-Hoc-Gruppen und andere kleinere Veranstaltungen statt, die so viel mit dem Thema des im öffentlichkeits(un)wirksamen Diskurs stets als ,sozial' etikettierten Zusammenhalts zu tun haben wie eine Hand mit einem Fledermausflügel: Sie entspringen zwar demselben Umstand, lösen aber andere Probleme. Während an der Oberfläche meist auf „natürliche Fragen“ (Luhmann 1997: 23) ebenso ,natürliche“ Antworten geliefert werden, wendet man sich in den Kellergeschossen vermehrt auch mit durchaus ,strengste[r] kritische[r] Einstellung“ (Bourdieu 1985: 67) dezidiert soziologischen Problemen zu, die sich vom ,idealen Schein“ einer vielfältig zusammenhaltenden Gesellschaft nicht blenden lassen und deshalb in ihren Antworten auf eine ganz andere Art und Weise überzeugen.

Ein bisschen erinnert das, was man in den fünf Tagen Bochum erlebt, sogar an den großen Newton: Dieser ist bekanntermaßen nicht nur systematischer Physiker gewesen, sondern auch leidenschaftlicher Alchemist. Hinsichtlich dieser Parallelisierung lässt sich aber eine eigentümliche Disproportionalität kaum verbergen. Gerade diejenige Praxis, die hier als KellerArbeit beschrieben wird, bescherte Newton nämlich öffentliche Aufmerksamkeit und nicht so sehr seine traditionsbewusste Alchemie ${ }^{1}$ (vgl. Wagner 2008: 151ff). Gerade umgekehrt scheint sich in Bochum die Soziologie auf der (zu Recht freilich kaum beachteten) Bühne als Alchemistin einer vielfältig-zusammenhaltenden Welt zu präsentieren, während sie ihre durchaus attraktive Seite als Verwalterin ,theorieabhängige[r] wissenschaftliche[r] Probleme“ (Luhmann 1997: 23) in den Kellern meint verstecken zu müssen. Vor dem Hintergrund dieser zweifelsfrei einseitigen Polung soll von dem Geschehen auf dem DGS-Kongress im Folgenden berichtet werden.

\section{Die Soziologie - Alchimistin einer besseren Welt?}

Ein Blick in die auffällig unauffälligen Zeitungsartikel, die sich mit dem Kongress beschäftigen, liefert sowohl dem gespannten Besucher als auch dem Nicht-Besucher den Eindruck, als ginge es bei einem solchen Kongress allen voran darum zu fragen, inwieweit Vielfalt zu begrüßen sei und ab welchem Grad Vielfalt zu Desintegration oder - wider Erwarten? - zu mehr Integration führe (Ruhr-Nachrichten 16.5.2012; Westdeutsche Allgemeine Zeitung 20.9.2012;

1 Die in fast wortwörtlicher Übersetzung des Kongressthemas auch Spagyrik heißt. 
Süddeutsche Zeitung 8.10.2012). ${ }^{2}$ In diesen Berichten ist dann üblicherweise die Rede von religiöser und ethnisch-kultureller Vielfalt, von familiärer und geschlechtlicher Vielfalt, von schichtungs- und milieubezogener Vielfalt, von lebensstilistischer und lebensförmlicher Vielfalt oder auch von Bildungs- und Erwerbsvielfalt.

Das Hauptprogramm mit seinen verschiedenen Vorreden verspricht nicht weniger eine Beleuchtung vor allem derjenigen Vielfalt, die uns als Teilnehmer am gesellschaftlichen Geschehen ohnehin jeden Tag in den Medien entgegenstrahlt. So wird etwa betont, das Ruhrgebiet sei ein optimaler Austragungsort für diesen Kongress, da man gerade hier an der Vielfalt nicht vorbeisehen könne. Eine solche Aussage verdeckt die nur ab und an gestellte Frage danach, was eigentlich unter Vielfalt verstanden werden soll bzw. welche Art von Vielfalt es von wem zu betrachten gilt. So wird zwar darauf verwiesen, es gehe auch darum, zu eruieren, was als Vielfalt wahrgenommen wird; dieser Satz muss aber vergessen werden, um die Information nachvollziehen zu können, dass sich anhand des Ruhrgebiets die „Ausdifferenzierung der Zusammensetzung der Wohnbevölkerung nach Herkunftsländern gut veranschaulichen [lasse]“ (Pries 2012: 9). Das ist sicher richtig, nur wird hier vorher schon gewusst, von welcher Art die gesuchte Vielfalt ist. Es drängt sich dem Leser der Einführungstexte ohnehin die Frage auf, was denn Nicht-Vielfalt sein soll. Wonach suchen wir auf diesem Kongress nicht? Wodurch grenzt er sich ab? Suchen wir nur nach bestimmten Arten von Vielfalt? Oberflächlich scheint es so. Der dritte Teil dieses Aufsatzes wird anhand der Erfahrungen im Keller des Kongresses aber zeigen, dass auf diesem keineswegs nur über das „Wechselverhältnis von Vielfalt und Zusammenhalt" (Pries / Meuser 2012: 16) gesprochen worden ist, sondern auch über Konzepte der Seiten dieser Unterscheidung selbst. Die gerade angesprochenen Unklarheiten sind wohl auch durch die „Vulgärbegriff[e]“ (Durkheim 1961: 119) ,Vielfalt" und ,Zusammenhalt" bedingt. ,Zusammenhalt' erweckt Vorstellungen von sich harmonisch die Hände reichenden Menschengruppen. Die in der verstaubten lateinischen Sprache (einstmals Garantin für den alltagsentfremdeten Gebrauch von Begriffen) gefasste Form ,Konsistenz ‘ weckte ganz andere Bilder und gerade Inkonsistenz wäre doch ebenfalls ein nicht zu bestreitendes Element von modernem Zusammenhalt. Will man Inkonsistenzen nicht einfach aus einer doch irgendwie zusammenhaltenden Gesellschaft verbannen, könnte man Vielfalt Einfalt gegenüberstellen. Dieser Gegenbegriffs-Kandidat kommt im Hauptprogramm aber nicht einmal vor. Dasselbe Schicksal trifft Kandidaten wie Allheit oder Einheit.

Wie es scheint, muss man erst einmal Rechenschaft über das Gemeinsame des soziologischen Subjekts ablegen - auch wenn dies ein etwas angestaubtes „Vorbild von Einheit“ (Adorno 2003: 18) ist. Diese Frage thematisiert konsequenterweise Gerhard Wagner in seiner Abendlesung Soziologie als Einzelwissenschaft: Eine Arbeitshypothese. Ausgehend von der Feststellung, die Soziologie sei eine vielfältig-multiparadigmatische Wissenschaft, mit deren Zusammenhalt es nicht so weit her sei, liefert Wagner eine Lösung für dieses Spannungsverhältnis. Sie besteht in einer Kritik der, romantischen“ Inkommensurabilitätserfahrung und der mit jener möglicherweise verbundenen Rehabilitierung des ,aufklärerischen' Reduktionsgedankens. Ob diese Forderung eines mit sich selbst identischen soziologischen Subjekts nebst einheitlicher Wissensbasis in unseren Fakultäten Plausibilität erhalten wird, kann man bezweifeln. Macht man aber aus der Not eine Tugend, könnte Subjektlosigkeit gerade die Stärke der Soziologie sein, auch wenn der Kongress doch bisweilen die Sorge aufkommen lässt, die Soziologie verwandele sich in Folge derselben zum Schamanen, der ,gleich den Kultmasken, die den vielen Geistern ähnlich sein sollten“ (Horkheimer / Adorno 2010: 15), seine Gestalt immerfort wechselt. Will man bloße Reduplikationen der ohnehin vorhandenen Selbstbe-

2 Zwei Artikel (Westdeutsche Allgemeine Zeitung, 4.10.2012, 8.10.2012) machen gar den Eindruck und es scheint nicht zufällig eine Nötigung für diese Art des Berichterstattens zu geben -, als handle es sich bei einem solchen Kongress um den alljährlichen Wandertag der Soziologenschaft. 
schreibungen verhindern, muss die Nichteinheitlichkeit der Soziologie nicht unbedingt reduziert werden. Gegenvorschläge könnten etwa darin bestehen, sie auszuhalten oder auszunutzen. Über all diese Alternativen zu streiten, würde sich jedenfalls lohnen - „das bloße Vermitteln wird nie produktiv weiter bringen“" (Heidegger 2010: 295).

Diese Konstellation im Blick habend, wird auch verständlich, warum von dem hier zu besprechenden Kongress gefordert wird, „Zusammenhalt innerhalb der soziologischen Profession [zu] erzeugen - durch die Vielfalt von und manchmal auch den Streit zwischen durchaus divergierenden, aber stets auf gemeinsame Frage- und Themenstellungen bezogenen Analysen und Befunden" (Pries / Meuser 2012: 25). Gerade der Kongress aber hat dem studentischen Beobachter nahegelegt, dass es nicht nur um verschiedene Antworten auf dieselben Fragen geht, sondern vor allem die Frage- und Themenstellungen so weit differieren, dass man selbst den Zusammenhalt einer solch überschaubaren Veranstaltung nicht erklären kann mit Hilfe von Normen, Konsens, Anerkennung oder was immer man sich als soziale Formen des Zusammenhalts ausdenken mag. ${ }^{3}$ Auch einen Streit über die Fragestellungen selbst sucht man vergeblich.

Zeitgleich zu dem anregenden Vortrag Wagners sprach Baskin Oran über Identity, Participation, and Cohesion in globalizing nation-states und stellte dabei vor allem die Türkei in den Vordergrund. Die Türkei - als Gastland des Kongresses - sei geradezu ein Experimentierfeld, auf dem der Sprung von einer Gesellschaftsstufe, deren Ideologie des Zusammenhalts die Nation sei, zu einer, deren Zentrum das Individuum bilde, nachvollzogen werden könne - der ,Turk' wird zum, Türkiyeli' ${ }^{`}$. Auch die Türkei gehe demnach den Weg von nationengebundener zu kultureller Bürgerschaft. Diese Ausführungen supponieren das - von Oran auch für den Kongress überhaupt proklamierte - Ausgangsproblem, ,how to build cohesion in a diversified society“. Orans Vortrag spiegelt damit geradezu die Außensicht, mit Hilfe derer die Soziologie sich in Bochum präsentiert. Es ist wohl ein glücklicher Zufall, dass diese beiden Vorträge zeitgleich stattgefunden haben, denn sie zeigen einen versteckten Zusammenhang auf. Gerade weil die Soziologie sich nicht auf einen Nenner bringen lässt und auch in ihren Grundbegriffen eine schillernde Vielfalt aufweist, verschafft sie sich ein in der Öffentlichkeit plausibles Label und erreicht damit gerade das Gegenteil von dem, was sie erreichen will. Und nicht zuletzt deshalb übernimmt sie wohl in einem so großen Maße die tagespolitischen und medialen Probleme (das zeigt nicht zuletzt der wieder aufgenommene Wahlkampf, in dem in keiner Rede das Wort ,Zusammenhalt' ausbleibt) und stülpt ihnen ein wissenschaftliches Vortragsformat über. Der Kongress muss die Spannung aushalten, der sich die Soziologie in eigentümlicher Weise ausgesetzt sieht. Es ist die Spannung des Stehens zwischen Öffentlichkeit und Forschungspragmatik. Dem Kongress ist die Überbrückung dieser Spannung mit Hilfe verschiedener Formate denn auch gelungen.

Der Vortrag von Renate Mayntz über die Finanzmarktkrise scheint ein Format bereitzustellen, das beide ,Interessen “ - das wissenschaftliche und das öffentlichkeitszentrierte - vereinigt. Hier ist jedenfalls nicht nur auf die Bedrohung der Sozialintegration (wachsende Vielfalt von Lebensentwürfen usw.), sondern auch auf die der Systemintegration hingewiesen worden. Von dem sichtbarsten Symptom letzterer - der Finanzmarktkrise - ausgehend greift Mayntz Mängel gängiger Differenzierungstheorien von Spencer bis Luhmann auf. Diese seien nämlich nicht in der Lage, die Ausdifferenzierung des Finanzsystems aus dem allzu „umfassend definierten“ Wirtschaftssystem mit all den Folgen, die sie zeitigt, in den Blick zu bekommen. Weil diese Theorien die Wirtschaft primär vom Geld (und nicht: von der Produktion) her denken, ent-

3 Die Absolution der Vielfalt lässt sich bezeichnenderweise auch dem Einführungstext selbst entnehmen, dessen Einheit nicht erkennbar ist. Dies gilt auch formal, denn sogar das Gründungsdatum der Universität Bochum ist vielfältig: man liest 1962 (Heinze / Pries / Boden 2012: 26) und 1963 (Pries / Schmidt / Boden 2012: 29). 
gleitet ihnen diese Möglichkeit. Damit liefert Renate Mayntz zum Zwecke der Erklärung ,handfester gesellschaftlicher' Probleme eine theorie-immanente Kritik (wie immer gerechtfertigt diese sein mag). Darüber hinausgehende Vorschläge betreffen letztlich nicht das politische Handeln selbst, sondern kreisen gerade um dessen Möglichkeitsbedingungen, die etwa im Falle einer tatsächlichen Ausdifferenzierung des Finanzsystems durch Abhängigkeitsverhältnisse eingeschränkt wären. Zu vermuten ist, dass der Applaus gerade wegen der Anerkennung dieses geglückten Spagats zwischen öffentlich brisantem Thema und Theoriefundiertheit nicht so recht enden wollte.

\section{Begriffsexperimente in den Kellergeschossen}

Für die Öffentlichkeitsarbeit stehen das Hauptprogramm und ein Großteil der Vorlesungen; hingegen erschließen sich dem Beobachter viele der Ad-Hoc-Gruppen und diverse Plenen nur mit Blick auf deren Forschungspragmatik. Hier werden andere Fragen gestellt als diejenigen, die auf ein politisch-öffentliches Publikum allzu gerecht zugeschnitten sind. So kann man etwa von einer Veranstaltung, die sich mit dem sinnhaften Ordnungsaufbau der Sozialwelt in ihren verschiedenen Sinn-Sphären beschäftigt (s.u.) direkt zu einem Vortrag hinüberwechseln, der die Unterscheidung von diversity und inequality in den Vordergrund stellt. Diese letztere von Göran Therborn hervorgekehrte Seite der vertikalen Vielfalt müsse nicht nur analytisch sauber gefasst, sondern auch sozial und politisch in den Fokus der Aufmerksamkeit gerückt werden - was immer dies konkret bedeuten soll. Therborn denkt bei diesbezüglichen institutional challenges jedenfalls an eine durchaus klassische Trias: Familie, bürgerliche Gesellschaft (Kapitalismus), Staat. Weiter verweist er als einer der wenigen auch auf die zunehmende Vereinheitlichung, die mit der Moderne einhergeht - auf Sprachen, Normen, Ideologien, Städteanordnungen, Essgewohnheiten, Sozialisations- und Bildungsformen.

Gerade wenn man die Differenz von großen und kleinen Veranstaltungen in den Blick nimmt, fällt auf, dass sich der Großteil der Vorlesungen - die Knotenpunkte eines solchen Kongresses und insofern ,Verbündete' der Öffentlichkeitsarbeit - einem Begriff von Vielfalt verschrieben hat, der den verschiedenen Arten der gesellschaftlichen Selbstbeschreibungen entnommen ist: Ethnische und religiöse Vielfalt ist das Thema Anna Triandafyllidous, die starken Wert darauf legt zu beachten, dass und weshalb manche Minderheiten und Arten von Ungleichheit mehr, andere hingegen weniger sichtbar seien; ethnische Vielfalt und ihre unterschiedliche Bewertung innerhalb von Städten ist auch das Thema Jan Raths - nur wer chic für den Chic produziert, scheint in Amsterdam legitimer Marktteilnehmer zu sein.

In den Kellergeschossen der Bochumer Ruhr-Universität trifft der studentische Kongressbesucher auch auf das, was sich der Einheit des Labels nicht unterwirft: Begriffsarbeit sowie Verweise auf zeitliche und sachliche Vielfalt. Hier geschieht damit auch das, was der Vortrag Hat die Soziologie einen sozialen Bias? von Armin Nassehi der Soziologie - neben öffentlichkeits(un)wirksamem Auftreten - abverlangt. Von dem in diesen Räumen Geschehenen sollen im Folgenden einige Impressionen gegeben werden.

In der Ad-Hoc Gruppe Die Zeitlichkeit des Sozialen etwa diagnostiziert die Organisatorin Maja Suderland das Fehlen einer geschichtsbewussten Perspektive in der gegenwärtigen Soziologie. Ein soziales Phänomen könne nur als Gewordenes angemessen erkannt werden. Dass dies eine Information ist, sorgte von Beginn an für etwas Verwunderung, wurde aber auch von den Vortragenden - etwa Robert Schmidt - bestärkt. Dieser konstatierte - ebenfalls zur Überraschung einiger Gasthörer -, die Soziologie habe es vor allem mit ,entzeitlichten' und ,statischen' theoretischen Modellen zu tun. Der Forschung entgehe so die Temporalität ihrer Gegenstände. Mit Hilfe von praxissoziologischen Mitteln aber auch mit Hilfe der Figurationssoziologie von Norbert Elias ließen sich diese Dimensionen aber einfangen. Im Anschluss daran sprach die Organisatorin selbst über Bourdieus temporales Denken und die Notwendigkeit, 
dessen prima facie räumlich konnotierten Begriffe auch in ihrer Zeitlichkeit fruchtbar zu machen. Der Habitus etwa verkörpere eine zunächst und zumeist vergessene Vergangenheit und eine in ihrem Möglichkeitsraum eingeschränkte Zukunft jeweils in der Gegenwart. Am Ende machte sie darüber hinaus den (noch nicht ausgearbeiteten) Vorschlag, die Zeitlichkeit als Klammer für die oft als lose gekoppelt deklarierte Theorieanlage Bourdieus anzusehen.

Im Anschluss entwickelte Gunter Weidenaus einen dreidimensionalen Zeitbegriff (Chronologie, Geschichte, Dauer), der nicht so sehr durch innovative Kraft, wohl aber durch Sauberkeit in der Analytik bestach. An diesen ließen sich beispielsweise Biografieentwürfe (episodisch, linear, zyklisch), aber auch Raumkonzeptionen koppeln. Wolfgang Knöbl stellte in seinen doch sehr bestimmten Ausführungen vor allem die Notwendigkeit in den Mittelpunkt, die vorhandenen Begriffe der Soziologie zu historisieren und zu kontextualisieren. Darüber hinaus warnte er vor einer allzu blinden Übernahme von hochumstrittenen Konzepten (wie dem der Sattelzeit) aus anderen Wissenschaften in die Soziologie. ${ }^{4}$ Nur mit Hilfe einer reflektierten Integration geschichtswissenschaftlicher und anthropologischer Mittel ließen sich soziologische Probleme heute noch lösen.

Das Plenum Soziale Differenzierungen, mannigfaltige Wirklichkeiten und kulturelle Orientierungsinstanzen war eine Veranstaltung, die sich explizit auch mit der sachlichen Dimension von Vielfalt beschäftigte, d.h. mit dem Nebeneinander verschiedener Wertsphären. Dieses von Joachim Fischer, Reiner Keller und Andreas Reckwitz organisierte Plenum begann mit einem Vortrag Uwe Schimanks, der von der Unumgänglichkeit dreier Topoi für jede Theorie der Moderne sprach: der funktionalen Differenzierung, einer marktvermittelten Ungleichheit und des Fortschritts als kulturell vermitteltem Ausblickspunkt. Er habe diese drei Stränge bei jedem Gesellschaftstheoretiker beobachtet, allerdings ließen diese meist den Zusammenhang der Dimensionen sowie die Kämpfe, die zwischen denselben stattfinden, unterbelichtet. Eine Theorie, die die Einheit dieser Dreiheit ernst nimmt, könne keine große Erzählung mehr sein, sondern nur mehr ,kontingente Bewegungen im Rahmen der Randbedingungen, die durch alle drei Ordnungen gesetzt werden' beschreiben. Achim Brosziewski konzentrierte sich auf Hierarchien von Differenzen. Man dürfe nicht nur die Gleichzeitigkeit verschiedener Praktiken sehen, sondern müsse auch beachten, dass es auf Seiten des Forschungsgegenstandes der Soziologie nach wie vor eine attraktive Option von Kritik sei, Soziales auf Hierarchien zu reduzieren. Nicht aber alle, sondern nur mehr Hierarchien zweiter Ordnung, d.h. Hierarchien zwischen mehreren asymmetrischen Differenzen, fänden auch in der Moderne Anschluss. Nach einer wissenssoziologischen Perspektive auf das Phänomen der Differenzierung durch Joachim Renn folgte der Vorschlag Anna Amelinas, den Begriff des Funktionssystems durch den des Ensembles zu ersetzen. Abschließend sprach Richard Münch über kulturelle Homogenisierung durch Pisa. Münch stellte keine Zunahme, sondern eine Abnahme von Vielfalt fest zumindest in Sachen Bildung. Nachdem der Zuhörer überzeugende - und trotz des Themas keineswegs normativ aufgeladene - Argumente dafür erhielt, dass PISA Gehorsam fördernde und Kritikfähigkeit stauchende Bildungssysteme besser einstuft, kritisierte auch Münch die unzulängliche Beachtung von Machtverschiebungen durch eine differenzierungstheoretische Herangehensweise. Entscheidend an PISA sei nämlich nicht zuletzt, dass die symbolische Macht von den Lehrern zu den Bildungsforschern abwandere.

Am Mittwochnachmittag wurde das anfangs schon erwähnte Buch Hans-Georg Soeffners diskutiert. Diese überaus gelungene Veranstaltung - von Ronald Hitzler nach eigener Aussage ohne Organisationsaufwand organisiert - nahm die sinnhafte Formung von Ordnungen und deren analytische Gliederung in den Blick. Hier sprachen Monika Wohlrab-Sahr, Helmuth

4 Ähnlich auch schon Horkheimer: „Es wird immer wieder hervorgehoben, daß dieselben Gegenstände in der einen Disziplin Probleme bilden, die in kaum absehbarer Zeit zu lösen sind, und in der anderen als schlichte Tatbestände hingenommen werden." (Horkheimer 1973: 21). 
Berking und Manfred Prisching. Erstere stellte wesentliche Inhalte dar und sogleich die dreifache Wirklichkeits-Sphären-Analytik Soeffners in Frage. Die erste Sphäre der unmittelbaren Interaktion sei nur eine Kontrastfolie, die es in der stets sinnhaft vermittelten Wirklichkeit nicht gäbe. Soeffner griff den in ihrem Argument verwendeten Begriff der Evidenz-Suggestion auf und stellte richtig, dass die erste Sphäre auch vermittelt sei - nur sich eben als unmittelbare aufdränge. Der Beitrag Helmuth Berkings rückte vor allem die oftmals mit Religiosität zusammenhängende Beschreibung in Soeffners Werk in den Mittelpunkt. Im Zuge der diesbezüglichen Antwort Soeffners, der den Unterschied von Religion und Religiosität stark machte, kam der interessante Gedanke zu Tage, die Grenze der Soziologie sei zugleich ihr Kern: das Individuum. Und als schließlich Manfred Prisching zur Illustration der Gedanken Hans-Georg Soeffners mit diesem auf der Leinwand einen Stuhl (genauer: den Wassily von Marcel Breuer) einkaufen ging, gab es auch noch etwas zu lachen.

Das Plenum Körperkulturen und kollektive Erregungen beschäftigte sich mit körperlichen Interaktionen und Eigendynamiken. Robert Seyfert warb etwa für ein ,Konzept der affektiven Interaktionen distributiver Körper und Akteure‘. Dafür führte er den Begriff des Affektifs ein, um sich allen voran gegen eine deterministische, statische und nur auf den Menschen beschränkte Betrachtung von Inter-Körperdynamiken abzugrenzen. Seyfert näherte sich so dem Phänomen der Inter-Körper-Beziehungen jenseits von Genetik, Triebstruktur, Semiotik und affektiver Atmosphäre. Nur so sei es möglich, aus der Interaktion hervorgehende Affekte zu erklären. Anschließend an diesen Vortrag beschäftigte sich Gabriele Klein mit Choreografien des Protests. Die kaum zu übersehende Ästhetisierung der Protest-Kultur führe nicht zu einer Entpolitisierung derselben, sondern zu einer Ästhetisierung des Politischen selbst. Als Einflussfaktoren für die Änderung der Protestkultur benannte sie vor allem die Änderung der Stadtformen. Auch Yvonne Niekrenz las über Kollektive - allerdings noch stärker in Bezug auf die Körperlichkeit derselben. Sie untersuchte deren Herstellung durch gemeinsame Bilder bzw. Imaginationen und Materialitäten wie Bekleidungen. Ronald Hitzler berichtete in diesem Plenum außerdem anhand des Unglücks auf der Love Parade in Duisburg von folgender empirisch zu beobachtender Kette von Kollektivzuständen: ersehnte kollektive Erregung - erlittene kollektive Erregung - Fassungslosigkeit - Trauer - Trauerbekundungen - Wut - Forderung von Verantwortung - letztendlicher moralischer Sieg über das vom Bürgermeister personifizierte moralisch Böse. Ferner stellten Thomas Scheffer und Robert Schmidt - nicht unbedingt für alle Anwesenden überzeugend - ihre Methode der Live-Soziologie vor. In einer auf offener Straße sich vollziehenden Manifestation des Politischen (genannt Protest) wird versucht, in actu die Auswirkungen auf den Involvierten zu beschreiben - zwar mit soziologischem tacit knowledge bewaffnet (und insofern: ,theoretisch'), aber stets qua Anschmiegen an den Gegenstand, der dann keiner mehr ist. ${ }^{5}$

Die Ad-Hoc Gruppe Konnektivität - Über die Logiken der Verbindung in der modernen Gesellschaft rückte vor allem die Begriffe Wiederholung, Operativität und Praxis in Bezug auf gesellschaftliche Differenzierungsprozesse und deren Niederschlag in unterschiedlichen Theorieangeboten in den Fokus der Betrachtung. Julian Müller machte in Anschluss an Tarde, Deleuze und Latour deutlich, dass Identitäten niemals etwas Primäres für sich, sondern stets auf ihre Relationierung in operation angewiesen seien. Wenn aber Strukturen von Symbolzusammenhängen Ergebnis ihrer Operationen sind (und sonst nichts), können auch angebliche Makrogebilde auf die Verkettung der Operationen, deren Form sie gemeinsam haben, reduziert werden. Hilmar Schäfer stellte ausgehend von einer praxistheoretischen Position dar, welche Probleme sich für die Theorie Bruno Latours ergäben. Diese rücke nur in einem unzureichen-

5 Und genau an dieser Stelle kommen einem wieder der Alchemist und der Schamane in den Sinn: „Die Zauberei ist wie die Wissenschaft auf Zwecke aus, aber sie verfolgt sie durch Mimesis, nicht in fortschreitender Distanz zum Objekt.“ (Horkheimer / Adorno 2010: 17). 
den Maße die inkorporierten Wissensbestände und die damit verbundene körperliche Widerstandsfähigkeit in den Fokus der Betrachtung. Ute Tellmann, Nicklas Baschek, Sven Opitz und Victoria von Groddeck führten Anwendungsfälle operativer Theorien vor - und zwar in Bezug auf die Themen Schulden, Politik, Menschenrechte und Diversity Management. Letzteres wird von der Vortragenden nicht primär hinsichtlich der Frage betrachtet, ob es tatsächlich zu erhöhter Produktivität oder Chancengleichheit führt; vielmehr wird die Frage gestellt, für welches gesellschaftliche Problem das Auftauchen von Diversity Management in Organisationen eine Lösung darstellt. Die Lösung besteht jedenfalls darin, für gleichzeitig höchst differenzierte und integrative Strukturen die Vermittlungsaufgabe zu übernehmen.

In diesen Veranstaltungen konnte man wertvolle Anregungen für die betreffenden Spezialgebiete und -themen sammeln. Für ausgiebige Diskussionen blieb allerdings keine Zeit - diese wurden (so meine naive Vermutung) erst im Anschluss an die Sitzungen auf den Gängen oder auch außerhalb des Kongresses geführt.

\section{Abschluss}

Neben der bisher entfalteten Spannung zwischen Bühnen- und Kellergeschossen verwunderten noch einige andere Dinge. Man vermisste doch - gerade an der sich als interdisziplinär ausflaggenden Ruhruniversität Bochum - die Auseinandersetzung mit anderen Wissenschaften. Bis auf die einleitenden Grußworte und Agnes Hellers philosophischem Abschluss waren keine nicht-soziologischen Stimmen zu vernehmen. Insbesondere ist es geradezu ein Versäumnis, das Vielfaltskonzept nicht ernster genommen und über evolutionstheoretische Konzepte nachgedacht zu haben; ist es doch die Biologie, die die Ordnung der Vielfalt schon seit 150 Jahren operationalisiert. Auch beschlich zumindest den studentischen Beobachter hin und wieder das Gefühl, dass die Soziologenschaft, was die Sache ihres Fachs betrifft, bisweilen nicht nur bei den ,Lorbeeren des bloßen Wollens' und ihren ,trockenen Blättern' stehenbleibt (,man sollte dies und jenes mehr berücksichtigen...'), sondern selbst dieses ,nichtige Wollen' schon preisgegeben hat - höchstwahrscheinlich eine Folge der kollektiv unbewussten Einsicht in die Notwendigkeit der Kontingenz allen Wissens. Merkwürdig ist dann aber - und auch diese Analogie folgt der eingangs erwähnten Buchdiskussion -, weshalb aus dem Messopfer trotzdem keine Messoper wird (vgl. Soeffner 2010: 48). Oder doch?

Der DGS-Kongress 2012 bleibt gerade aufgrund dieser widersprüchlichen Eindrücke durchaus als ein merk-würdiges Ereignis im Gedächtnis. Man lernte - so zumindest die ,bourdieusche' Hoffnung des Textverfassers - nicht nur explizit und inhaltlich einiges dazu. So etwa, dass gewisse Streitthemen, die auf dem Kongress in Tübingen vor gut 50 Jahren noch die Gemüter erhitzt haben, heute zum Großteil - ohne ausgetragen zu sein - schlicht der Indifferenz und wissenschaftlichen Arbeitsteilung zugefallen sind. Auch verwundert es, dass gerade die Soziologie sich so sehr vom common-sense gängeln lässt, da sie doch um ihre Funktion fürchten muss, wenn sie sich nicht klar von ohnehin schon im Umlauf befindlichen Semantiken abgrenzt. Hier sollte jedenfalls gezeigt werden, inwiefern man sich im Laufe der Woche ein klareres Bild davon machen konnte, weshalb sich ein Fach für einen Kongress solch einen seltsamen (den ein oder anderen auch abschreckenden) Titel gibt, der zudem eine unscharfe und regredierende Wiederholung des Themas von 1996 in Dresden (Differenz und Integration) darstellt. Zusammenhalt durch labeling. Gleichwohl verschwindet durch dieses Label das unter ihm subsumierte Nichtidentische nicht. Insofern ist keineswegs ausgemacht, dass die Formel ,Vielfalt und Zusammenhalt‘ als Symptom für einen Rückfall der Soziologie in vorsoziologische Zustände gelesen werden muss. Vielleicht wird im Gegenteil erst durch diese Spannung sichtbar, dass es gute Gründe gibt, die öffentliche Unsichtbarkeit des Kongresses in einen Ansporn für die Bergung der Praktiken in den Kellergeschossen umzumünzen. 


\section{Literaturverzeichnis}

Adorno, Theodor W. (2003): Zur Metakritik der Erkenntnistheorie. Studien über Husserl und die phänomenologischen Antinomien, in: Ders., Gesammelte Schriften, hrsg. v. Rolf Tiedemann, Band 5, Frankfurt / Main, S. 7-245.

Bourdieu, Pierre (1985): Leçon sur la leçon, in: Ders., Sozialer Raum und ,Klassen’. Leçon sur la leçon. Zwei Vorlesungen, Frankfurt / Main, S. 49-81.

Durkheim, Emile (1961): Die Regeln der soziologischen Methode, Neuwied.

Heidegger, Martin (2010): Kant und das Problem der Metaphysik. Frankfurt / Main.

Heinze, Rolf / Ludger Pries / Sven Boden (2012): Das Ruhrgebiet - Metropole im Umbruch, in: Hauptprogramm des 36. Kongresses der Deutschen Gesellschaft für Soziologie, S. 25-28.

Horkheimer, Max (1973): Traditionelle und kritische Theorie, in: Ders., Traditionelle und kritische Theorie. Vier Aufsätze, Frankfurt / Main, S. 12-56.

Horkheimer, Max / Theodor W. Adorno (2010): Dialektik der Aufklärung. Philosophische Fragmente, Frankfurt / Main.

Luhmann, Niklas (1997): Die Gesellschaft der Gesellschaft. Zwei Bände, Frankfurt / Main.

Pries, Ludger (2012): Vorwort des Sprechers des Organisationskomitees, in: Hauptprogramm des 36. Kongresses der Deutschen Gesellschaft für Soziologie, S. 8-13.

Pries, Ludger / Michael Meuser (2012): Vielfalt und Zusammenhalt - 36. Kongress der Deutschen Gesellschaft für Soziologie, in: Hauptprogramm des 36. Kongresses der Deutschen Gesellschaft für Soziologie, S. 14-25.

Pries, Ludger / Stefanie Schmidt / Sven Boden (2012): Sozialwissenschaft und Soziologie an der RUB, in: Hauptprogramm des 36. Kongresses der Deutschen Gesellschaft für Soziologie, S. 29-30.

Ruhr-Nachrichten (16.5.2012): Soziologie-Stars reisen an, Bericht von Max Florian Kühlem, abrufbar unter: http://www.dgs2012.de/wp-content/uploads/Presse/16.05.RN.pdf, letztes Abrufdatum: 15.3.2013.

Soeffner, Hans-Georg (2010): Symbolische Formung. Eine Soziologie des Symbols und des Rituals, Weilerswist.

Süddeutsche Zeitung (8.10.2012): Das Leben entlang der A40. Beim 36. Kongress der Deutschen Gesellschaft für Soziologie ging es um Migration, Religion und die Türkei, Bericht von Volker Breidecker, S. 13.

Wagner, Lioba (2008): Vielfalt in der Wissenschaft - Der Beitrag der Alchemie zur Naturwissenschaft bei Paracelsus, Boyle und Newton, Dissertation an der Universität Trier, abrufbar unter: http:// ubt.opus.hbz-nrw.de/volltexte/2008/489/pdf/Vielfalt_in_der_Wissenschaft.pdf, letztes Abrufdatum: 8.10.2012.

Westdeutsche Allgemeine Zeitung (20.9.2012): Haben wir zu viel Vielfalt? Arme und Reiche, Christen und Muslime, Migranten und Einheimische: Forscher untersuchen, wie viele Gegensätze unsere Gemeinschaft aushält, Bericht von Christopher Onkelbach, abrufbar unter: http://www.dgs2012.de/wpcontent/uploads/WAZ-Kongress-Artikel.pdf, letztes Abrufdatum: 15.3.2013.

Westdeutsche Allgemeine Zeitung (4.10.2012): Wissenschaft trifft Wirklichkeit. Soziologen besuchen Brennpunkte - eine Fahrt in Dortmunds Nordstadt, Bericht von Christopher Onkelbach, abrufbar unter: http://www.dgs2012.de/wp-content/uploads/Presse/04.10.WAZ.pdf, letztes Abrufdatum: 15.3.2013.

Westdeutsche Allgemeine Zeitung (8.10.2010): Ausflug ins wirkliche Leben. Soziologen verlassen Hörsaal, um in Marxloh Herausforderungen zu erkunden, Bericht von Miriam Lenkeit, S. 21, abrufbar unter: http://www.dgs2012.de/wp-content/uploads/Presse/08.10.WAZ.pdf, letztes Abrufdatum: 15.3.2013. 
Kurt Georg Rachlitz

Ludwig-Maximilians-Universität München

Institut für Soziologie

Konradstraße 6

80801 München

kurt.rachlitz@soziologie.uni-muenchen.de 